Review Article

\title{
Involvement of lncRNAs and Macrophages: Potential Regulatory Link to Angiogenesis
}

\author{
Yang Jia $\mathbb{D}^{1}$ and Yedi Zhou $\mathbb{D}^{2,3}$ \\ ${ }^{1}$ Department of Pediatrics, The Second Xiangya Hospital, Central South University, Changsha, Hunan 410011, China \\ ${ }^{2}$ Department of Ophthalmology, The Second Xiangya Hospital, Central South University, Changsha, Hunan 410011, China \\ ${ }^{3}$ Hunan Clinical Research Center of Ophthalmic Disease, Changsha, Hunan 410011, China
}

Correspondence should be addressed to Yedi Zhou; zhouyedi@csu.edu.cn

Received 1 November 2019; Revised 29 January 2020; Accepted 4 February 2020; Published 29 February 2020

Guest Editor: Mohammad A. Khan

Copyright () 2020 Yang Jia and Yedi Zhou. This is an open access article distributed under the Creative Commons Attribution License, which permits unrestricted use, distribution, and reproduction in any medium, provided the original work is properly cited.

\begin{abstract}
Macrophages are involved in angiogenesis, an essential process for organ growth and tissue repair, and could contribute to the pathogenesis of angiogenesis-related diseases such as malignant tumors and diabetic retinopathy. Recently, long noncoding RNAs (lncRNAs) have been proved to be important in cell differentiation, organismal development, and various diseases of pathological angiogenesis. Moreover, it has been indicated that numerous lncRNAs exhibit different functions in macrophage infiltration and polarization and regulate the secretion of inflammatory cytokines released by macrophages. Therefore, the focus of macrophage-related lncRNAs could be considered to be a potential method in therapeutic targeting angiogenesis-related diseases. This review mainly summarizes the roles played by lncRNAs which associated with macrophages in angiogenesis. The possible mechanisms of the regulatory link between lncRNAs and macrophages in various angiogenesis-related diseases were also discussed.
\end{abstract}

\section{Introduction}

Angiogenesis is the growth process of blood vessels and plays important roles in the physiological functions for organ growth and tissue repair [1], as well as a large number of angiogenesis-related diseases such as tumors, arthritis, diabetic retinopathy, and age-related macular degeneration [2]. Targeting angiogenesis is an effective therapeutic method for anticancer treatment and has been applied in many kinds of cancer (e.g., lung cancer [3, 4] and gastric cancer [5]). The treatment of antivascular endothelial growth factor (VEGF) has been applied in inhibiting angiogenesis, especially in cancer [6] and ocular diseases [7]. However, beyond VEGF, there are also a variety of other molecules that play important roles in the mechanisms of angiogenesis $[8,9]$.

Long noncoding RNAs (lncRNAs) are those which demonstrate no apparent protein-coding capacity and longer than 200 nucleotides [10]. Recent studies indicated a variety of regulatory functions of lncRNAs in a wide range of cellular and developmental processes as well as pathogenesis [11-15].
In particular, lncRNAs control cell differentiation and selfrenewal through neural, skin, and muscle stem cells [16]. LncRNAs are also involved in diseases of pathological angiogenesis, such as diabetic retinopathy $[17,18]$.

Macrophages are important angiogenic effector cells and act as key modulators in both tumor growth and angiogenesis [19]. Many studies suggested that under various stimuli, macrophages could be polarized to two phenotypes: classically activated M1 phenotype and alternatively activated M2 phenotype [20-22]. Those M1 macrophages can destroy foreign organisms and inhibit tumor growth, while M2 phenotype functions in wound healing, chronic infections, tumor growth, and angiogenesis [23-29]. We previously revealed that M2 macrophages, rather than M1 phenotype, infiltrated in the inner layer of the retinas of oxygeninduced retinopathy and enhanced retinal neovascularization in vivo [30]. In a choroidal neovascularization mouse model, we recognized that M1 and M2 macrophages have different distributions, thus might have diverse potential biological functions in angiogenesis [31]. A recent study 
reported that lncRNA MM2P regulated tumorigenesis and angiogenesis via modulating M2-like macrophage polarization [32], indicating that lncRNAs and macrophages might be involved and have a potential regulatory link to angiogenesis.

In the present review, we summarize the roles of lncRNAs associated with macrophages in angiogenesis and discuss the possible mechanisms of the regulatory link between lncRNAs and macrophages in various angiogenesis-related diseases.

\section{LncRNAs Regulate Macrophage Infiltration, Polarization, and Functions}

Monocytes are considered as the precursors of macrophages, originated from hematopoietic stem cells, and monocyte/macrophage differentiation plays a critical role in response to the immune system and pathological diseases [33-35]. It has been indicated that IncRNA lnc-MC was involved in monocyte/macrophage differentiation, positively regulated by PU.1, a hematopoiesis-specific transcription factor, and negatively interacted with miR-199a to promote differentiation process [36]. Besides monocyte/macrophage differentiation, lncRNAs seemed to be involved in macrophage infiltration. For example, downregulation of LRNA9884 significantly suppressed macrophage infiltration by reducing the level of monocyte chemoattractant protein-1 (MCP-1) in a type 2 diabetic nephropathy mice model [37]. Moreover, lncRNA CASC2c could inhibit macrophage migration and M2 polarization by negatively regulating the expression of coagulation factor $\mathrm{X}$, which was reported to promote the infiltration of macrophages to the glioblastoma multiforme tumor cells, and polarize macrophages to M2 phenotype [38]. In contrast, activated lncRNA UCA1 promoted macrophage infiltration, resulting in carcinogenesis and progression of breast cancer [39].

LncRNAs could also induce macrophage polarization and lead to regulatory effects on their functions. Lipopolysaccharide (LPS) and interleukin- (IL-) 4 induction was commonly applied for M1/M2 macrophage polarization, respectively [32]. Ye et al. observed that lncRNA Cox-2 is expressed higher in LPS-induced M1 macrophages than IL-4-induced M2 macrophages, and silencing lncRNA Cox-2 expression markedly altered the macrophage polarization from M1 to M2 phenotype [40]. In addition, lncRNA Cox-2 siRNA significantly enhanced the ability of macrophages in tumor proliferation, invasion, and migration by mediating M1/M2 polarization [40]. Moreover, using the gain-of-function and loss-of-function strategies, lncRNA TUC339 was recognized to be required for macrophage polarization to regulate the release of pro- or antiinflammatory cytokines and thereby affect tumor growth [41]. Overexpression of TUC339 in hepatocellular carcinoma (HCC) cells suppressed the expression of proinflammatory factors, such as IL- $1 \beta$ and TNF- $\alpha$, and knockdown of TUC339 obtained an opposite effect [41]. It has been reported that LPS could strengthen the lncRNA CCL2 levels to mediate the expressions of inflammatory factors in macrophages, and this enhancement could be suppressed by SIRT1 in sepsis [42]. Knockdown of lncRNA CCL2 resulted in a reduction of IL- $1 \beta$, IL- 6 , and TNF- $\alpha$ [42]. In response to
LPS, lncRNA Nfkb2 and lncRNA Rel, located near proinflammatory transcription genes, were increased and closely related to the inflammatory response in mouse macrophages [43].

Some lncRNAs could target related molecules or signaling pathways to regulate macrophage polarization. For example, IncRNA GAS5 was significantly reduced in M2-polarized microglia, and overexpression of GAS5 suppressed microglial M2 polarization via inhibition of transcription of IRF4, which is an important regulatory molecule of M2 polarization [44]. As we discussed, lncRNA MM2P was higher expressed in M2 macrophages rather than in M1 macrophages, and blockade of lncRNA MM2P could weaken the IL-4/STAT6 signaling pathway, resulting in a reduction of both cytokine-regulated M2 polarization and M2-induced angiogenesis [32]. NF- $\kappa \mathrm{B}$, which is a downstream signaling pathway of toll-like receptors (TLRs) after specific microbial and pathogen recognition, could induce transcription of proinflammatory genes and is strongly involved in the regulation of macrophage polarization [45]. After LPS stimulation, the expression of lncRNA Mirt2 was induced in macrophages and suppressed the proinflammatory factors (such as TNF, IL-1 $\beta$, IL-6, and IL-12) by inhibiting the activation of NF- $\kappa \mathrm{B}$ and MAPK pathways [46]. In contrast to LPS, Mirt2 also could promote the polarization of M2 macrophages induced by IL-4, but the mechanism might be independent from STAT6 and PPAR $\gamma$ pathways [46]. Under LPS-mediated inflammatory conditions, lncRNA Tnfaip3 exerts a coregulatory role with NF- $\kappa \mathrm{B}$ in modulating inflammatory gene transcription in macrophages [47]. Another NF- $\kappa$ B-mediated lncRNA FIRRE exhibited posttranscriptional elevation of inflammatory genes in macrophages and epithelial cells by interacting with heterogeneous nuclear ribonucleoproteins U after LPS stimulating [48]. Overall, these studies showed that the expression profiles of lncRNAs can be clearly distinguished between M1 and M2 macrophages, indicating that lncRNAs could be involved in regulating macrophage polarization. Dysregulation of lncRNAs may affect macrophage polarization by targeting both downstream signaling pathways and the release of inflammation cytokines.

According to competing endogenous RNA (ceRNA) networks, lncRNAs could act as sponges to regulate the functions of miRNAs [49]. Studies had demonstrated that lncRNA NIFK-AS1 and lncRNA CCAT1 could inhibit the polarization of M2 macrophages by targeting miR146a and miR-148a, respectively [50, 51]. Moreover, lncRNA XIST and IncRNA GNAS-AS1 exhibited the promotion of M2 polarization, such functions were associated with T-cellspecific transcription factor 4 (TCF-4) and miR-4319, respectively $[52,53]$.

MALAT1 is an important lncRNA that has been widely investigated [18, 54-57]. Recent studies had reported that the MALAT1 regulates the production of inflammatory cytokines [56] and was increased in a LPS-induced acute lung injury model to regulate the release of IL- $1 \beta$, IL- 6 , and TNF- $\alpha$ [58]. Silencing of MALAT1 inhibited the proinflammatory responses by enhancing miR-146a levels in macrophages and epithelial cells [58]. In LPS-induced septic cardiomyocytes, expression of MALAT1 was induced by 


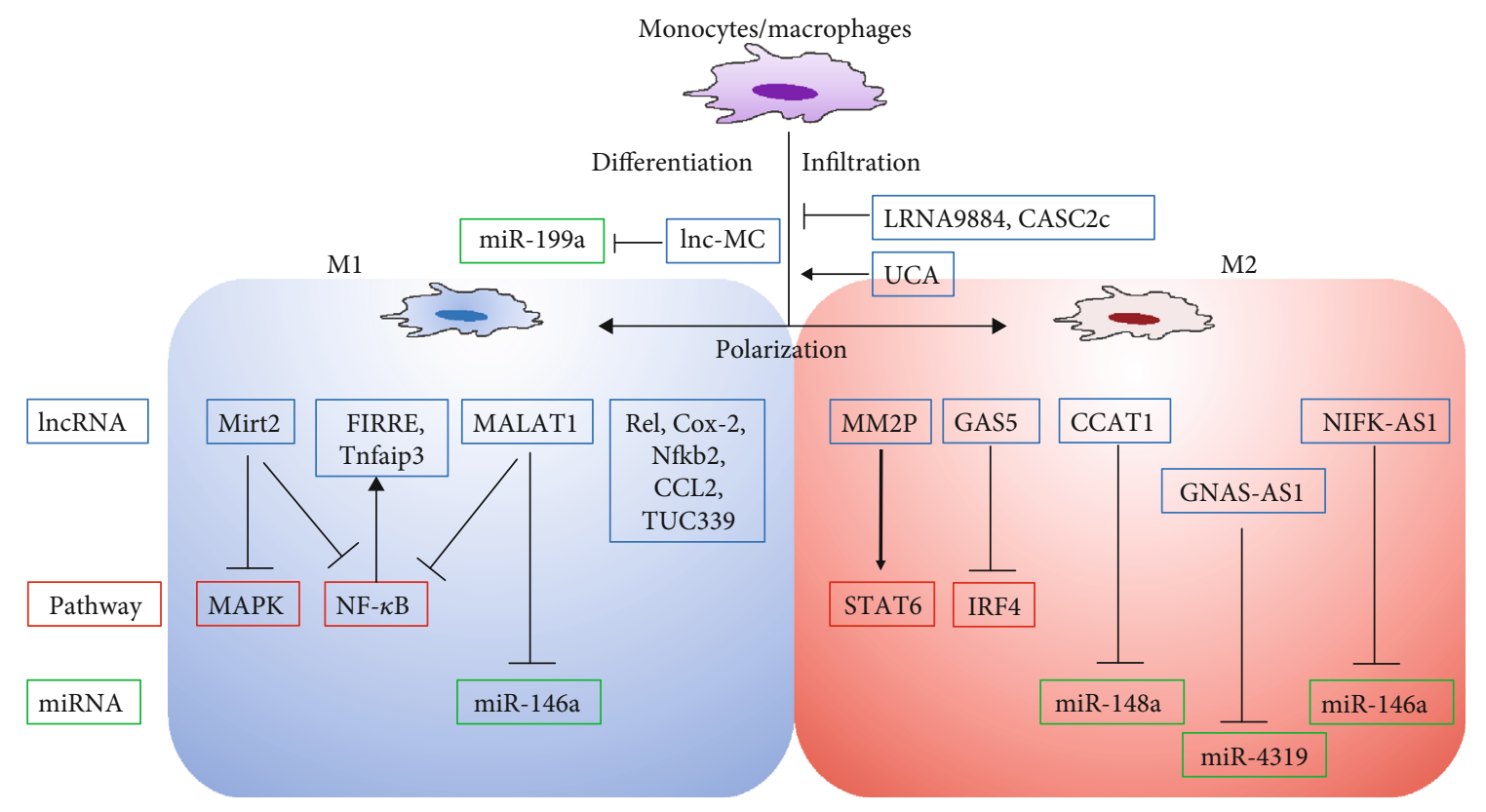

FIGURE 1: The mechanisms of lncRNAs regulate macrophage infiltration, polarization, and functions.

IL- 6 and elevated the production of TNF- $\alpha$ partially through serum amyloid antigen 3 (SAA3) [59]. By targeting SAA3, MALAT1 also could modulate the expression of IL- 6 and TNF- $\alpha$ in the endothelial cells under high-glucose conditions [60]. Although the proinflammatory activities of MALAT1 in macrophages were reported, Zhao et al. presented an opposite effect of MALAT1, which functions as an antiinflammatory regulation in vitro [61]. In this study, scientists had demonstrated that MALAT1 was upregulated by LPS to suppress the production of proinflammatory TNF- $\alpha$ and IL- 6 by interacting with the NF- $\kappa \mathrm{B}$ pathway in macrophages. The knockdown of MALAT1 achieved enhancement of TNF- $\alpha$ and IL-6 [61]. It is known that tumor-associated macrophages (TAMs) exhibit similar functions to M2 macrophages [62] and MALAT1 was upregulated in TAMs compared to nonpolarized macrophages and promoted angiogenesis through secretion of fibroblast growth factor-2 (FGF2) protein [63]. Moreover, in macrophages, MALAT1 regulates lysosomal-associated membrane protein 1 (lamp1) expression by sponging miR-23-3p [64]. For the above contradictory effect of MALAT1 in inflammatory responses, further investigations are required to reveal the essential mechanisms of MALAT1 to macrophage functions in angiogenesis.

Together, the above studies suggest that lncRNAs could regulate macrophage infiltration, polarization, inflammation, and secretion by targeting various pathways to change the pro- and/or anti-inflammatory response mechanisms (Figure 1). Further studies on the mechanism of lncRNAs in macrophages can lead to enhance the understanding on how lncRNAs might be involved in inflammation and thereby affect the regulation of immune response of angiogenesis.

\section{Link between Macrophages and Angiogenesis}

It is widely considered that M1 macrophages present a proinflammatory effect and M2 macrophages present an anti- inflammatory effect. Besides, M2 macrophages also induce proangiogenic functions, and the induction of M2 macrophages enhances cancer invasion and metastasis, as well as the development of neovascular diseases through VEGF [65-67]. By now, although the activation of the downstream pathway during angiogenesis is still not completely clear, activated macrophages could influence the angiogenic process through the production of angiogenic factors such as IL-1, IL-6, IL- 8 , TNF- $\alpha$, TGF- $\alpha$, TGF- $\beta$, GM-CSF, bFGF, and VEGF [68]. Moreover, activation of NF- $\kappa$ B and STAT3 is involved in the upstream pathway of macrophageinduced angiogenesis $[69,70]$. Thus, macrophages and angiogenesis are very closely linked with complicated mechanisms.

\section{Involvement of lncRNAs in the Pathogenesis of Angiogenesis- Related Diseases}

4.1. Tumor Angiogenesis. Many studies revealed the involvement of lncRNAs in the recruitment of macrophages to tumor cells and M1/M2 polarization of macrophages to change the tumor microenvironment.

As we discussed before, MALAT1 not only acted as a potential cancer biomarker [54] but also regulated angiogenesis in diabetic retinopathy [71], tumor [63, 72-74], hindlimb ischemia [75], and brain vascular endothelium [76]. In particular, as we described, Huang et al. reported that MALAT1 enhanced thyroid cancer angiogenesis by regulating FGF2 secretion of TAMs [63]. In HCC cells, MALAT1 could promote angiogenesis and regulate polarization of macrophages through sponging miR-140 [74]. These suggested that macrophages might be an important modulator of angiogenesis in the mechanisms of MALAT1. 
As mentioned above, MM2P could contribute to promoting M2 polarization of macrophages and inducing angiogenesis, resulting in tumor deterioration [32]. As we described, lncRNA UCA1 was demonstrated to be involved in macrophage recruitment to promote breast cancer invasion in a previous study [39]. In cervical cancer cells, UCA1 was upexpressed and negatively associated with miR-206, and knockdown of UCA1 directly decreased VEGF through miR-206 upregulation, and thereby suppressed tumor growth, viability, migration, and invasion [77]. Another lncRNA TUC339 was significantly increased in cancer stem cell-derived exosomes, and VEGF was enhanced in exosomes derived from cancer stem cells correspondingly [78]. The knockdown of TUC339 reduced HCC cell growth and spread [79]. The mechanism has been uncovered that TUC339 could regulate the macrophage polarization, functioning as promotion of anti-inflammatory cytokines and angiogenesis, thereby accelerating tumor proliferation [41]. Sang et al. showed that lncRNA CamK-A was involved in macrophage infiltration and angiogenesis by triggering the transcription of the $\mathrm{NF}-\kappa \mathrm{B}$ signaling pathway in tumor cells [80]. By promoting NF- $\kappa$ B downstream cytokines (e.g., VEGF, IL-6, and TNF- $\alpha$ ), Camk-A could remodel tumor microenvironment to recruit macrophages to tumors and contribute to angiogenesis [80]. LncRNA LNMAT1 upregulated CCL2 and recruited M2 macrophages to the tumor, and promoted lymphatic metastasis via excretion of VEGF-C [81]. These studies indicated that lncRNAs could regulate the recruitment of macrophages to the tumor, macrophage polarization, secretion of VEGF, and thereby the induction of pathological angiogenesis and tumor growth and spread.

It has been demonstrated that lncRNA PVT1 is involved in the high microvessel density in gastric cancer as well as the promotion of tumor growth through activation of the STAT3 signaling pathway as well as secretion of VEGFA [82]. The knockdown of lncRNA ROR was reported to reduce angiogenesis through inhibition of NF- $\kappa$ B and JAK1/STAT3 pathways [83]. Moreover, overexpression of miR-26 could rescue the negative effects of ROR silencing, demonstrating that ROR functions as a molecular sponge for miR-26 in these activations [83]. LncRNA LIMT was suppressed by epidermal growth factor (EGF) and downregulated in breast cancer and ovarian cancer, and the EGF secreted from TAMs suppressed the levels of LIMT through activation of the EGF-ERK pathway [84, 85]. Although the direct links between these lncRNAs and macrophages were poorly indicated, it is possible that lncRNAs could interact with macrophage-related signaling pathways to regulate the tumor angiogenesis.

4.2. Angiogenesis in Other Diseases. Many major causes for blindness, such as age-related macular degeneration, retinopathy of prematurity, diabetic retinopathy, and retinal vein occlusions, are due to the pathological angiogenesis [86]. In particular, diabetic retinopathy, a complication of diabetes mellitus, is a major cause of blindness worldwide in which pathological processes are characterized by the formation of abnormal blood vessels within the eye [87]. LncRNAs could target macrophage-related signaling pathways to regulate the pathological angiogenesis. With the high-glucose treatment in human retinal endothelial cells, the expression of lncRNA ANRIL was increased and regulated VEGF expression through polycomb repressive complex 2 (PRC2) complex [88]. By binding to the NF- $\kappa \mathrm{B}$ signaling pathway, ANRIL could induce pathologic damage of retinopathy in the diabetic rat model [89]. Moreover, ANRIL could also promote angiogenesis by activating the NF- $\kappa$ B pathway in diabetes combined with cerebral infraction in a rat model [90]. Similarly, the expression of lncRNA MIAT was also elevated on high glucose stress through impacting the VEGF signaling pathway, while knockdown of MIAT attenuated retinal vessel dysfunction [91]. Clinical investigations in diabetes patients had shown that increased expression of MIAT was markedly associated with diabetic retinopathy process, and the increased MIAT decreased the viability of ARPE-19 cells in vitro via targeting the TGF- $\beta 1$ pathway [92]. The highglucose conditions suppress the expression of lncRNA MEG3, whereas the rescue of MEG3 could delay diabetic retinopathy by inhibiting TGF-1 and VEGF levels [93]. In addition, MEG3 could also be regulated by activation of the PI3k/Akt pathway in diabetes mellitus-related microvascular dysfunction [94].

LncRNA NEAT1 was reported to be involved in M2 macrophage polarization [95] and could promote inflammation in macrophages $[96,97]$. NEAT1 could accelerate angiogenesis by enhancing VEGF, SIRT1, and BCL-XL in brain microvascular endothelial cells [98]. Indeed, loss of NEAT1 expression exhibits downregulation of VEGF and upregulation of miR-377 resulting in antiangiogenesis and proapoptosis [98], while the mechanisms of macrophage polarization and functions lack investigation. In contrast, lncRNA MEG3 negatively regulated angiogenesis after ischemic stroke via suppressing the Notch pathway [99], and the silencing of MEG3 resulted in a proangiogenesis effect in vascular endothelial cells [100]. Yan et al. found MEG3 could be activated and participated in apoptosis of macrophages under oxidized low-density lipoprotein stimulation, indicating a novel role of MEG3/miR-204/CDKN2A pathway in macrophages [101]. Therefore, these two lncRNAs were reported to be related to both angiogenesis and macrophages in each study, and it is highly hypothesized that lncRNAs might alter macrophage functions to regulate pathological angiogenesis. On the other hand, we demonstrated that M2 macrophages, rather than M1, have essential functions in promoting retinal pathological neovasculization, while more experimental evidence is needed to support this hypothesis [30]. In our previous study, 198 upregulated and 175 downregulated IncRNAs were identified by microarray analysis in an oxygen-induced retinopathy mouse model [102]. Among them, we highlighted four validated lncRNAs that could be potentially involved in cell adhesion molecules and thereby affect the progress of pathological retinal angiogenesis [102]. In a mouse model of choroidal neovascularization induced by laser photocoagulation, we identified 716 altered lncRNAs, and the altered target genes of 7 validated lncRNAs were enriched in the immune system process and the chemokine signaling pathway [103]. Therefore, macrophages might also be involved in the immunological regulation associated with those altered lncRNAs. 


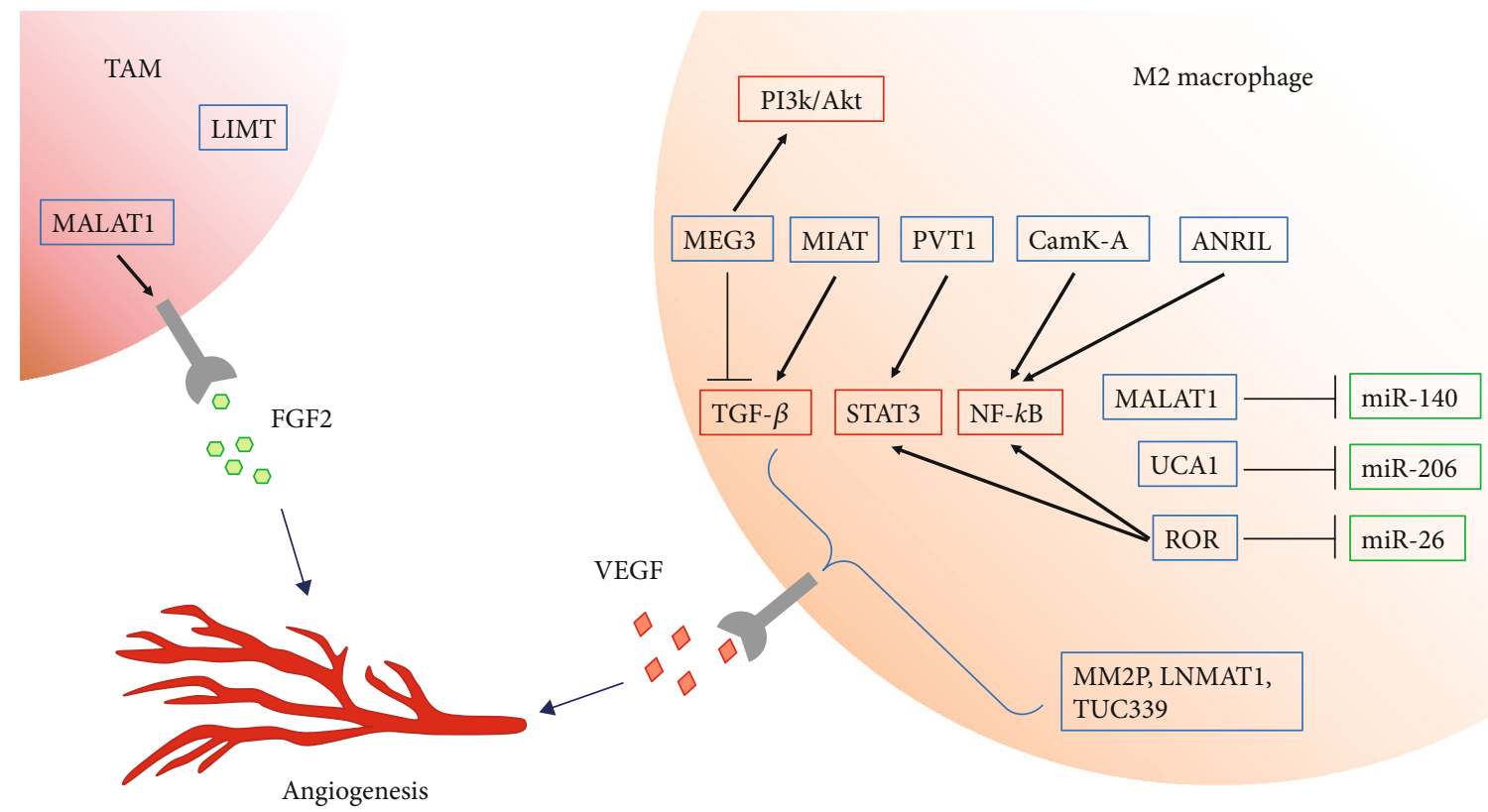

FIGURE 2: lncRNAs target macrophage-related pathways or miRNAs in macrophages to induce angiogenesis via elevating expression of VEGF or FGF2 in angiogenesis-related diseases.

Moreover, lncRNA could be involved in monocyte/macrophage differentiation to regulate the pathogenesis. For example, lncRNA NTT was reported to be elevated in rheumatoid arthritis and its activation contributes to monocyte/macrophage differentiation, resulting in the pathological process of rheumatoid arthritis [104].

Thus, IncRNAs are involved in various diseases associated with angiogenesis (Figure 2 ) and partially via the regulation of the functions of macrophages.

\section{Summary}

In sum, IncRNAs have been proved to play essential roles in angiogenesis in a variety of diseases. As shown in Figure 2, the mechanisms of direct effect to endothelial cells include regulating the secretion of growth factors or cytokines, such as VEGF or FGF2, and through a diverse range of pathways. On the other hand, some lncRNAs may also be associated with macrophage infiltration, differentiation, and polarization, and both lncRNAs and macrophages were involved in and have potential links to angiogenesis. Though some progress has been achieved in characterizing the functional lncRNAs in regulation of macrophage polarization, the mechanisms remain unclear, and further investigations are needed to understand the exact roles of lncRNAs which link to macrophages and angiogenesis. Therefore, targeting lncRNAs and the links with macrophages could be considered a novel therapeutic method in treating angiogenesis in different diseases.

\section{Conflicts of Interest}

The authors report no conflicts of interest.

\section{Acknowledgments}

This work was supported by the National Natural Science Foundation of China (No. 81800855), Hunan Province Natural Science Foundation (No. 2018JJ3765), Changsha Science and Technology Project (kq1907075), and Hunan Department of Science and Technology (No. 2015TP2007).

\section{References}

[1] P. Carmeliet, "Angiogenesis in life, disease and medicine," Nature, vol. 438, no. 7070, pp. 932-936, 2005.

[2] A. Fallah, A. Sadeghinia, H. Kahroba et al., "Therapeutic targeting of angiogenesis molecular pathways in angiogenesis- dependent diseases," Biomedicine \& Pharmacotherapy, vol. 110, pp. 775-785, 2019.

[3] S. M. Lee, P. Baas, and H. Wakelee, "Anti-angiogenesis drugs in lung cancer,” Respirology, vol. 15, no. 3, pp. 387-392, 2010.

[4] A. Onn, J. Bar, and R. S. Herbst, "Angiogenesis inhibition and lung-cancer therapy," The Lancet Oncology, vol. 15, no. 2, pp. 124-125, 2014.

[5] M. Zang, L. Hu, B. Zhang et al., "Luteolin suppresses angiogenesis and vasculogenic mimicry formation through inhibiting Notch1-VEGF signaling in gastric cancer," Biochemical and Biophysical Research Communications, vol. 490, no. 3, pp. 913-919, 2017.

[6] N. Ferrara, K. J. Hillan, H. P. Gerber, and W. Novotny, "Discovery and development of bevacizumab, an anti-VEGF antibody for treating cancer," Nature Reviews Drug Discovery, vol. 3, no. 5, pp. 391-400, 2004.

[7] P. Osaadon, X. J. Fagan, T. Lifshitz, and J. Levy, "A review of anti-VEGF agents for proliferative diabetic retinopathy," Eye, vol. 28, no. 5, pp. 510-520, 2014.

[8] T. Nakama, S. Yoshida, K. Ishikawa et al., "Inhibition of choroidal fibrovascular membrane formation by new class of 
RNA interference therapeutic agent targeting periostin," Gene Therapy, vol. 22, no. 2, pp. 127-137, 2015.

[9] Y. Kobayashi, S. Yoshida, Y. Zhou et al., "Tenascin-C secreted by transdifferentiated retinal pigment epithelial cells promotes choroidal neovascularization via integrin alphaV," Laboratory Investigation, vol. 96, no. 11, pp. 1178-1188, 2016.

[10] G. Bohmdorfer and A. T. Wierzbicki, "Control of chromatin structure by long noncoding RNA," Trends in Cell Biology, vol. 25, no. 10, pp. 623-632, 2015.

[11] N. A. Rapicavoli, K. Qu, J. Zhang, M. Mikhail, R. M. Laberge, and H. Y. Chang, "A mammalian pseudogene lncRNA at the interface of inflammation and anti-inflammatory therapeutics," eLife, vol. 2, article e00762, 2013.

[12] L. Yang, C. Lin, C. Jin et al., "IncRNA-dependent mechanisms of androgen-receptor-regulated gene activation programs," Nature, vol. 500, no. 7464, pp. 598-602, 2013.

[13] X. Sun, Y. Yuan, Y. Xiao et al., "Long non-coding RNA, $B m c o b$, regulates osteoblastic differentiation of bone marrow mesenchymal stem cells," Biochemical and Biophysical Research Communications, vol. 506, no. 3, pp. 536-542, 2018.

[14] K. Liu, H. Yao, Y. Wen et al., "Functional role of a long noncoding RNA LIFR-AS1/miR-29a/TNFAIP3 axis in colorectal cancer resistance to pohotodynamic therapy," Biochimica et Biophysica Acta (BBA) - Molecular Basis of Disease, vol. 1864, no. 9, pp. 2871-2880, 2018.

[15] J. Jia, M. Zhang, Q. Li, Q. Zhou, and Y. Jiang, "Long noncoding ribonucleic acid NKILA induces the endoplasmic reticulum stress/autophagy pathway and inhibits the nuclear factor-k-gene binding pathway in rats after intracerebral hemorrhage," Journal of Cellular Physiology, vol. 233, no. 11, pp. 8839-8849, 2018.

[16] R. A. Flynn and H. Y. Chang, "Long noncoding RNAs in cellfate programming and reprogramming," Cell Stem Cell, vol. 14, no. 6, pp. 752-761, 2014.

[17] B. Yan, Z. F. Tao, X. M. Li, H. Zhang, J. Yao, and Q. Jiang, "Aberrant expression of long noncoding RNAs in early diabetic retinopathy," Investigative Ophthalmology \& Visual Science, vol. 55, no. 2, pp. 941-951, 2014.

[18] S. Biswas, A. A. Thomas, S. Chen et al., "MALAT1: an epigenetic regulator of inflammation in diabetic retinopathy," Scientific Reports, vol. 8, no. 1, p. 6526, 2018.

[19] C. Lamagna, M. Aurrand-Lions, and B. A. Imhof, "Dual role of macrophages in tumor growth and angiogenesis," Journal of Leukocyte Biology, vol. 80, no. 4, pp. 705-713, 2006.

[20] M. Hesketh, K. B. Sahin, Z. E. West, and R. Z. Murray, "Macrophage phenotypes regulate scar formation and chronic wound healing," International Journal of Molecular Sciences, vol. 18, no. 7, article 1545, 2017.

[21] A. Mantovani, A. Sica, S. Sozzani, P. Allavena, A. Vecchi, and M. Locati, "The chemokine system in diverse forms of macrophage activation and polarization," Trends in Immunology, vol. 25, no. 12, pp. 677-686, 2004.

[22] M. Locati, A. Mantovani, and A. Sica, "Macrophage activation and polarization as an adaptive component of innate immunity," Development and Function of Myeloid Subsets, vol. 120, pp. 163-184, 2013.

[23] S. Gordon and F. O. Martinez, "Alternative activation of macrophages: mechanism and functions," Immunity, vol. 32, no. 5, pp. 593-604, 2010.
[24] S. Nakao, K. Noda, S. Zandi et al., "VAP-1-mediated M2 macrophage infiltration underlies IL- $1 \beta$ - but not VEGF-Ainduced lymph- and angiogenesis," The American Journal of Pathology, vol. 178, no. 4, pp. 1913-1921, 2011.

[25] S. Zandi, S. Nakao, K. H. Chun et al., "ROCK-isoformspecific polarization of macrophages associated with agerelated macular degeneration," Cell Reports, vol. 10, no. 7, pp. 1173-1186, 2015.

[26] B. M. Delavary, W. M. van der Veer, M. van Egmond, F. B. Niessen, and R. H. J. Beelen, "Macrophages in skin injury and repair," Immunobiology, vol. 216, no. 7, pp. 753-762, 2011.

[27] A. Mantovani, T. Schioppa, C. Porta, P. Allavena, and A. Sica, "Role of tumor-associated macrophages in tumor progression and invasion," Cancer Metastasis Reviews, vol. 25, no. 3, pp. 315-322, 2006.

[28] V. W. H. Ho and L. M. Sly, "Derivation and characterization of murine alternatively activated (M2) macrophages," Methods in Molecular Biology, vol. 531, pp. 173-185, 2009.

[29] N. Jetten, S. Verbruggen, M. J. Gijbels, M. J. Post, M. P. J. de Winther, and M. M. P. C. Donners, "Anti-inflammatory M2, but not pro-inflammatory M1 macrophages promote angiogenesis in vivo," Angiogenesis, vol. 17, no. 1, pp. 109-118, 2014.

[30] Y. Zhou, S. Yoshida, S. Nakao et al., "M2 macrophages enhance pathological neovascularization in the mouse model of oxygen-induced retinopathy," Investigative Ophthalmology \& Visual Science, vol. 56, no. 8, pp. 4767-4777, 2015.

[31] Y. Zhou, S. Yoshida, Y. Kubo et al., "Different distributions of M1 and M2 macrophages in a mouse model of laser-induced choroidal neovascularization," Molecular Medicine Reports, vol. 15, no. 6, pp. 3949-3956, 2017.

[32] J. Cao, R. Dong, L. Jiang et al., "LncRNA-MM2P identified as a modulator of macrophage M2 polarization," Cancer Immunology Research, vol. 7, no. 2, pp. 292-305, 2019.

[33] F. Geissmann, "Development of monocytes, macrophages, and dendritic cells (vol 327, pg 656, 2010)," Science, vol. 330, no. 6009, pp. 1318-1318, 2010.

[34] C. Shi and E. G. Pamer, "Monocyte recruitment during infection and inflammation," Nature Reviews Immunology, vol. 11, no. 11, pp. 762-774, 2011.

[35] C. Auffray, M. H. Sieweke, and F. Geissmann, "Blood monocytes: development, heterogeneity, and relationship with dendritic cells," Annual Review of Immunology, vol. 27, pp. 669-692, 2009.

[36] M. T. Chen, H. S. Lin, C. Shen et al., "PU.1-regulated long noncoding RNA lnc-MC controls human monocyte/macrophage differentiation through interaction with MicroRNA 199a-5p," Molecular and Cellular Biology, vol. 35, no. 18, pp. 3212-3224, 2015.

[37] Y. Y. Zhang, P. M. K. Tang, P. C. T. Tang et al., "LRNA9884, a novel Smad3-dependent long noncoding RNA, promotes diabetic kidney injury indb/dbMice via enhancing MCP-1dependent renal inflammation," Diabetes, vol. 68, no. 7, pp. 1485-1498, 2019.

[38] Y. Zhang, J. Feng, H. Fu et al., "Coagulation factor X regulated by $\mathrm{CASC} 2 \mathrm{c}$ recruited macrophages and induced M2 polarization in glioblastoma multiforme," Frontiers in Immunology, vol. 9, 2018.

[39] S. Chen, C. Shao, M. Xu et al., "Macrophage infiltration promotes invasiveness of breast cancer cells via activating long 
non-coding RNA UCA1," International Journal of Clinical and Experimental Pathology, vol. 8, no. 8, pp. 9052-9061, 2015.

[40] Y. Ye, Y. Xu, Y. Lai et al., "Long non-coding RNA cox-2 prevents immune evasion and metastasis of hepatocellular carcinoma by altering M1/M2 macrophage polarization," Journal of Cellular Biochemistry, vol. 119, no. 3, pp. 29512963, 2018.

[41] X. Li, Y. Lei, M. Wu, and N. Li, "Regulation of macrophage activation and polarization by HCC-derived exosomal IncRNA TUC339," International Journal of Molecular Sciences, vol. 19, no. 10, article 2958, 2018.

[42] Y. Jia, Z. Li, W. Cai et al., "SIRT1 regulates inflammation response of macrophages in sepsis mediated by long noncoding RNA," Biochimica et Biophysica Acta-Molecular Basis of Disease, vol. 1864, no. 3, pp. 784-792, 2018.

[43] A.-P. Mao, J. Shen, and Z. Zuo, "Expression and regulation of long noncoding RNAs in TLR4 signaling in mouse macrophages," BMC Genomics, vol. 16, no. 1, article 1270, 2015.

[44] D. Sun, Z. Yu, X. Fang et al., "LncRNA GAS5 inhibits microglial M2 polarization and exacerbates demyelination," EMBO Reports, vol. 18, no. 10, pp. 1801-1816, 2017.

[45] N. Wang, H. Liang, and K. Zen, "Molecular mechanisms that influence the macrophage M1-M2 polarization balance," Frontiers in Immunology, vol. 5, p. 9, 2014.

[46] M. Du, L. Yuan, X. Tan et al., "The LPS-inducible lncRNA Mirt2 is a negative regulator of inflammation," Nature Communications, vol. 8, no. 1, p. 2049, 2017.

[47] S. Ma, Z. Ming, A. Y. Gong et al., "A long noncoding RNA, lincRNA-Tnfaip3, acts as a coregulator of NF- $\kappa$ B to modulate inflammatory gene transcription in mouse macrophages," The FASEB Journal, vol. 31, no. 3, pp. 1215-1225, 2017.

[48] Y. Lu, X. Liu, M. Xie et al., “The NF- $\kappa$ B-Responsive long noncoding RNA FIRRE regulates posttranscriptional regulation of inflammatory gene expression through interacting with hnRNPU," Journal of Immunology, vol. 199, no. 10, pp. 3571-3582, 2017.

[49] L. Salmena, L. Poliseno, Y. Tay, L. Kats, and P. P. Pandolf, "A ceRNA hypothesis: the Rosetta Stone of a hidden RNA language?," Cell, vol. 146, no. 3, pp. 353-358, 2011.

[50] Y. X. Zhou, W. Zhao, L. W. Mao et al., "Long non-coding RNA NIFK-AS1 inhibits M2 polarization of macrophages in endometrial cancer through targeting miR-146a," International Journal of Biochemistry \& Cell Biology, vol. 104, pp. 25-33, 2018.

[51] J. Liu, D. Ding, Z. Jiang, T. du, J. Liu, and Z. Kong, "Long noncoding RNA CCAT1/miR-148a/PKC zeta prevents cell migration of prostate cancer by altering macrophage polarization," Prostate, vol. 79, no. 1, pp. 105-112, 2019.

[52] Y. Sun and J. Xu, "TCF-4 regulated lncRNA-XIST promotes M2 polarization of macrophages and is associated with lung cancer," Oncotargets and Therapy, vol. 12, pp. 8055-8062, 2019.

[53] Z. Li, C. Feng, J. Guo, X. Hu, and D. Xie, "GNAS-AS1/miR4319/NECAB3 axis promotes migration and invasion of non-small cell lung cancer cells by altering macrophage polarization," Functional \& Integrative Genomics, vol. 20, no. 1, pp. 17-28, 2020.

[54] Z. X. Li, Q. N. Zhu, H. B. Zhang, Y. Hu, G. Wang, and Y. S. Zhu, "MALAT1: a potential biomarker in cancer," Cancer Management and Research, vol. 10, pp. 6757-6768, 2018.
[55] M. Zhao, S. Wang, Q. Li, Q. Ji, P. Guo, and X. Liu, "MALAT1: a long non-coding RNA highly associated with human cancers (Review)," Oncology Letters, vol. 16, no. 1, pp. 19-26, 2018.

[56] L. E. Abdulle, J. L. Hao, O. P. Pant et al., "MALAT1 as a diagnostic and therapeutic target in diabetes-related complications: a promising long-noncoding RNA," International Journal of Medical Sciences, vol. 16, no. 4, pp. 548-555, 2019.

[57] T. Gutschner, M. Hammerle, and S. Diederichs, "MALAT1- a paradigm for long noncoding RNA function in cancer," Journal of Molecular Medicine, vol. 91, no. 7, pp. 791-801, 2013.

[58] L. Dai, G. Zhang, Z. Cheng et al., "Knockdown of LncRNA MALAT1 contributes to the suppression of inflammatory responses by up-regulating miR-146a in LPS-induced acute lung injury," Connective Tissue Research, vol. 59, no. 6, pp. 581-592, 2018.

[59] Y. T. Zhuang, D. Y. Xu, G. Y. Wang, J. L. Sun, Y. Huang, and S. Z. Wang, "IL-6 induced lncRNA MALAT1 enhances TNF- $\alpha$ expression in LPS-induced septic cardiomyocytes via activation of SAA3," European Review for Medical and Pharmacological Sciences, vol. 21, no. 2, pp. 302-309, 2017.

[60] P. Puthanveetil, S. Chen, B. Feng, A. Gautam, and S. Chakrabarti, "Long non-coding RNA MALAT1 regulates hyperglycaemia induced inflammatory process in the endothelial cells," Journal of Cellular and Molecular Medicine, vol. 19, no. 6, pp. 1418-1425, 2015.

[61] G. Zhao, Z. Su, D. Song, Y. Mao, and X. Mao, "The long noncoding RNA MALAT1 regulates the lipopolysaccharideinduced inflammatory response through its interaction with NF- $\kappa$ B," FEBS Letters, vol. 590, no. 17, pp. 2884-2895, 2016.

[62] S. Ramanathan and N. Jagannathan, "Tumor associated macrophage: a review on the phenotypes, traits and functions," Iranian Journal of Cancer Prevention, vol. 7, no. 1, pp. 1-8, 2014.

[63] J. K. Huang, L. Ma, W. H. Song et al., "LncRNA-MALAT1 promotes angiogenesis of thyroid cancer by modulating tumor-associated macrophage FGF2 protein secretion," Journal of Cellular Biochemistry, vol. 118, no. 12, pp. 4821-4830, 2017.

[64] Z. Ma, J. Zhang, X. Xu et al., "LncRNA expression profile during autophagy and Malat1 function in macrophages," PLoS One, vol. 14, no. 8, article e221104, 2019.

[65] D. Hanahan and R. A. Weinberg, "Hallmarks of cancer: the next generation," Cell, vol. 144, no. 5, pp. 646-674, 2011.

[66] A. Sica, P. Larghi, A. Mancino et al., "Macrophage polarization in tumour progression," Seminars in Cancer Biology, vol. 18, no. 5, pp. 349-355, 2008.

[67] M. Du, K. M. Roy, L. Zhong, Z. Shen, H. E. Meyers, and R. C. Nichols, "VEGF gene expression is regulated posttranscriptionally in macrophages," FEBS Journal, vol. 273, no. 4, pp. 732-745, 2006.

[68] C. Sunderkötter, K. Steinbrink, M. Goebeler, R. Bhardwaj, and C. Sorg, "Macrophages and angiogenesis," Journal of Leukocyte Biology, vol. 55, no. 3, pp. 410-422, 1994.

[69] K. H. Seo, H. M. Ko, J. H. Choi et al., "Essential role for platelet-activating factor-induced NF- $\kappa \mathrm{B}$ activation in macrophage-derived angiogenesis," European Journal of Immunology, vol. 34, no. 8, pp. 2129-2137, 2004.

[70] M. Ramirez-Pedraza and M. Fernandez, "Interplay between macrophages and angiogenesis: a double-edged sword in liver disease," Frontiers in Immunology, vol. 10, 2019. 
[71] P. Liu, S. B. Jia, J. M. Shi et al., "LncRNA-MALAT1 promotes neovascularization in diabetic retinopathy through regulating miR-125b/VE-cadherin axis," Bioscience Reports, vol. 39, no. 5, 2019.

[72] A. E. Tee, B. Liu, R. Song et al., "The long noncoding RNA MALAT1 promotes tumor-driven angiogenesis by upregulating pro-angiogenic gene expression," Oncotarget, vol. 7, no. 8, pp. 8663-8675, 2016.

[73] X. J. Huang, Y. Xia, G. F. He et al., "MALAT1 promotes angiogenesis of breast cancer," Oncology Reports, vol. 40, no. 5, pp. 2683-2689, 2018.

[74] Z.-H. Hou, X. W. Xu, X. Y. Fu, L. D. Zhou, S. P. Liu, and D. M. Tan, "Long non-coding RNA MALAT1 promotes angiogenesis and immunosuppressive properties of HCC cells by sponging miR-140," American Journal of Physiology-Cell Physiology, 2019.

[75] X. Zhang, X. Tang, M. Hamblin, and K. J. Yin, "Long noncoding RNA Malat1 regulates angiogenesis in hindlimb ischemia," International Journal of Molecular Sciences, vol. 19, no. 6, p. 1723, 2018.

[76] L. Ren, C. Wei, K. Li, and Z. Lu, "LncRNA MALAT1 up-regulates VEGF-A and ANGPT2 to promote angiogenesis in brain microvascular endothelial cells against oxygenglucose deprivation via targetting miR-145," Bioscience Reports, vol. 39, no. 3, 2019.

[77] Q. Yan, Y. Tian, and F. Hao, "Downregulation of lncRNA UCA1 inhibits proliferation and invasion of cervical cancer cells through miR-206 expression," Oncology Research, 2018.

[78] F. A. Alzahrani, M. A. el-Magd, A. Abdelfattah-Hassan et al., "Potential effect of exosomes derived from cancer stem cells and MSCs on progression of DEN-induced HCC in rats," Stem Cells International, vol. 2018, Article ID 8058979, 17 pages, 2018.

[79] T. Kogure, I. K. Yan, W. L. Lin, and T. Patel, "Extracellular vesicle-mediated transfer of a novel long noncoding RNA TUC339: a mechanism of intercellular signaling in human hepatocellular cancer," Genes \& Cancer, vol. 4, no. 7-8, pp. 261-272, 2013.

[80] L. Sang, H. Q. Ju, G. P. Liu et al., "LncRNA CamK-A regulates $\mathrm{Ca}^{2+}$-signaling-mediated tumor microenvironment remodeling," Molecular Cell, vol. 72, no. 1, pp. 71-83.e7, 2018.

[81] C. Chen, W. He, J. Huang et al., "LNMAT1 promotes lymphatic metastasis of bladder cancer via CCL2 dependent macrophage recruitment," Nature Communications, vol. 9, no. 1, article 3826, 2018.

[82] J. Zhao, P. du, P. Cui et al., "LncRNA PVT1 promotes angiogenesis via activating the STAT3/VEGFA axis in gastric cancer," Oncogene, vol. 37, no. 30, pp. 4094-4109, 2018.

[83] W. W. Qin, Z. L. Xin, H. Q. Wang, K. P. Wang, X. Y. Li, and $\mathrm{X}$. Wang, "Inhibiting lncRNA ROR suppresses growth, migration and angiogenesis in microvascular endothelial cells by up-regulating miR-26," European Review for Medical and Pharmacological Sciences, vol. 22, no. 22, pp. 7985-7993, 2018.

[84] A. Sas-Chen, M. R. Aure, L. Leibovich et al., "LIMT is a novel metastasis inhibiting lncRNA suppressed by EGF and downregulated in aggressive breast cancer," EMBO Molecular Medicine, vol. 8, no. 9, pp. 1052-1064, 2016.

[85] X. Y. Zeng, H. Xie, J. Yuan et al., "M2-like tumor-associated macrophages-secreted EGF promotes epithelial ovarian cancer metastasis via activating EGFR-ERK signaling and suppressing lncRNA LIMT expression," Cancer Biology \& Therapy, vol. 20, no. 7, pp. 956-966, 2019.

[86] A. Yoshida, S. Yoshida, T. Ishibashi, and H. Inomata, "Intraocular neovascularization," Histology and Histopathology, vol. 14, no. 4, pp. 1287-1294, 1999.

[87] N. Cheung, P. Mitchell, and T. Y. Wong, "Diabetic retinopathy," The Lancet, vol. 376, no. 9735, pp. 124-136, 2010.

[88] A. A. Thomas, B. Feng, and S. Chakrabarti, "ANRIL: a regulator of VEGF in diabetic retinopathy," Investigative Ophthalmology \& Visual Science, vol. 58, no. 1, pp. 470-480, 2017.

[89] J. C. Wei, Y. L. Shi, and Q. Wang, "LncRNA ANRIL knockdown ameliorates retinopathy in diabetic rats by inhibiting the NF- $\kappa$ B pathway," European Review for Medical and Pharmacological Sciences, vol. 23, no. 18, pp. 7732-7739, 2019.

[90] B. Zhang, D. Wang, T. F. Ji, L. Shi, and J. L. Yu, "Overexpression of lncRNA ANRIL up-regulates VEGF expression and promotes angiogenesis of diabetes mellitus combined with cerebral infarction by activating NF- $\kappa \mathrm{B}$ signaling pathway in a rat model," Oncotarget, vol. 8, no. 10, pp. 17347-17359, 2017.

[91] B. Yan, J. Yao, J. Y. Liu et al., "IncRNA-MIAT regulates microvascular dysfunction by functioning as a competing endogenous RNA," Circulation Research, vol. 116, no. 7, pp. 1143-1156, 2015.

[92] Q. Li, L. Pang, W. Yang, X. Liu, G. Su, and Y. Dong, "Long non-coding RNA of myocardial infarction associated transcript (LncRNA-MIAT) promotes diabetic retinopathy by upregulating transforming growth factor- $\beta 1$ (TGF- $\beta 1$ ) signaling," Medical Science Monitor, vol. 24, pp. 9497-9503, 2018.

[93] D. Zhang, H. Qin, Y. Leng et al., "LncRNA MEG3 overexpression inhibits the development of diabetic retinopathy by regulating TGF- $\beta 1$ and VEGF," Experimental and Therapeutic Medicine, vol. 16, no. 3, pp. 2337-2342, 2018.

[94] G.-Z. Qiu, W. Tian, H. T. Fu, C. P. Li, and B. Liu, "Long noncoding RNA-MEG3 is involved in diabetes mellitus-related microvascular dysfunction," Biochemical and Biophysical Research Communications, vol. 471, no. 1, pp. 135-141, 2016.

[95] Y. Gao, P. Fang, W. J. Li et al., "LncRNA NEAT1 sponges miR-214 to regulate $\mathrm{M} 2$ macrophage polarization by regulation of B7-H3 in multiple myeloma," Molecular Immunology, vol. 117, pp. 20-28, 2020.

[96] P. Zhang, L. Cao, R. Zhou, X. Yang, and M. Wu, "The lncRNA Neat1 promotes activation of inflammasomes in macrophages," Nature Communications, vol. 10, no. 1, article 1495, 2019.

[97] D. D. Chen, L. L. Hui, X. C. Zhang, and Q. Chang, "NEAT1 contributes to ox-LDL-induced inflammation and oxidative stress in macrophages through inhibiting miR-128," Journal of Cellular Biochemistry, vol. 120, no. 2, pp. 2493-2501, 2019.

[98] Z. W. Zhou, L. J. Zheng, X. Ren, A. P. Li, and W. S. Zhou, "LncRNA NEAT1 facilitates survival and angiogenesis in oxygen-glucose deprivation (OGD)-induced brain microvascular endothelial cells (BMECs) via targeting miR-377 and upregulating SIRT1, VEGFA, and BCL-XL," Brain Research, vol. 1707, pp. 90-98, 2019.

[99] J. Liu, Q. Li, K. S. Zhang et al., "Downregulation of the long non-coding RNA Meg3 promotes angiogenesis after ischemic brain injury by activating notch signaling," Molecular Neurobiology, vol. 54, no. 10, pp. 8179-8190, 2017. 
[100] C. He, W. Yang, J. Yang et al., "Long noncoding RNA MEG3 negatively regulates proliferation and angiogenesis in vascular endothelial cells," DNA and Cell Biology, vol. 36, no. 6, pp. 475-481, 2017.

[101] L. Yan, Z. Liu, H. Yin, Z. Guo, and Q. Luo, "Silencing of MEG3 inhibited ox-LDL-induced inflammation and apoptosis in macrophages via modulation of the MEG3/miR204/CDKN2A regulatory axis," Cell Biology International, vol. 43, no. 4, pp. 409-420, 2019.

[102] L. Zhang, X. Fu, H. Zeng et al., "Microarray analysis of long non-coding RNAs and messenger RNAs in a mouse model of oxygen-induced retinopathy," International Journal of Medical Sciences, vol. 16, no. 4, pp. 537-547, 2019.

[103] L. Zhang, H. Zeng, J. H. Wang et al., "Altered long noncoding RNAs involved in immunological regulation and associated with choroidal neovascularization in mice," International Journal of Medical Sciences, vol. 17, no. 3, pp. 292-301, 2020.

[104] C. A. Yang, J. P. Li, J. C. Yen et al., "lncRNA NTT/PBOV1 axis promotes monocyte differentiation and is elevated in rheumatoid arthritis," International Journal of Molecular Sciences, vol. 19, no. 9, article 2806, 2018. 